\title{
Escova dentária: a escolha do consumidor
}

Toothbrush: the choice of the consumer

Cepillo de dientes: la elección del consumidor

Tânia Adas SALIBA

Lia Borges de Mattos CUSTODIO

Lourenço Vieira Tereza CANEVARI

Nemre Adas SALIBA

Suzely Adas Saliba MOIMAZ

Departamento de Odontologia Preventiva e Restauradora - UNESP, Universidade Estadual Paulista

Faculdade de Odontologia de Araçatuba 16015-050 Araçatuba-SP, Brasil

\section{Resumo}

A higiene bucal, parte integrante dos cuidados corporais, é realizada com o auxílio de dispositivos, em especial, a escova dental. Com a evolução tecnológica, as escovas dentais passaram a ser fabricadas com diferentes materiais. Objetivou-se investigar as marcas e modelos de escovas dentárias disponíveis no mercado brasileiro; analisar a legislação vigente sobre a produção e verificar as informações fornecidas pelo fabricante ao consumidor final. Trata-se de um estudo transversal, descritivo, qualitativo, de análise documental. Foi realizado levantamento nas páginas eletrônicas da Agencia Nacional de Vigilância Sanitária e do Instituto Nacional de Metrologia, Qualidade e Tecnologia sobre a legislação vigente, que estabelece as características exigidas para produção e comercialização de escovas dentárias. Foram pesquisadas marcas e modelos disponíveis no mercado brasileiro, nas páginas eletrônicas das empresas, fabricantes ou importadoras e em pontos de vendas para o consumidor final para verificar as informações disponíveis nas embalagens. A Resolução $\mathrm{N}^{\circ}$ 54/17 da Diretoria Colegiada da ANVISA, estabelece algumas características físicas, tais como: largura máxima da cabeça e comprimento mínimo da escova dentária. Foram incluídos nesta pesquisa 20 marcas comerciais, as quais distribuem 108 modelos no mercado brasileiro. Apenas 2 modelos (1,85\%) apresentaram todas as informações exigidas pela resolução da ANVISA, sendo a "supervisão do adulto" e "orientação do dentista" os itens menos contemplados. Não apresentaram indicação para uso adulto ou infantil $44,4 \%$ do total. Conclui-se, que existem muitas marcas e modelos no mercado brasileiro, porém faltam informações importantes para o profissional realizar a indicação apropriada e o paciente, a aquisição consciente.

Descritores: Escovação Dentária; Higiene Bucal; Saúde Bucal.

\section{Abstract}

Oral hygiene, an integral part of body care, is performed with the aid of devices, especially the dental brush. With the technological evolution, the dental brushes began to be manufactured with different materials, varied types, formats and sizes. The objective of this research was to investigate the brands and models of toothbrushes available in the Brazilian market; review existing legislation on production as well as verify the information provided by the manufacturer to the final consumer. This is a cross-sectional, descriptive, qualitative study of documentary analysis. A survey was carried out on the websites of the National Sanitary Surveillance Agency and the National Institute of Metrology, Quality and Technology on the current legislation, which establishes the characteristics required for the production and commercialization of toothbrushes. We researched brands and models available in the Brazilian market, on the websites of companies, manufacturers or importers and at points of sales for the final consumer to collect information on the packaging. Resolution No. 54/17 of the Collegiate Board of ANVISA, establishes some physical characteristics, such as: maximum head width and minimum toothbrush length. 20 commercial brands were included in the survey, which distributed 108 models in the Brazilian market. Only 2 models (1.85\%) presented all the information required by the ANVISA resolution, with "adult supervision" and "dentist orientation" being the least contemplated items. There was no indication for adult or child use, $44.4 \%$ of the total. It is concluded that there are many brands and models in the Brazilian market but important information is missing for the professional to make the appropriate indication and the patient, the conscious acquisition. Descriptors: Toothbrushing; Oral Hygiene; Oral Health.

\section{Resumen}

La higiene bucal, parte integral del cuidado corporal, se realiza con la ayuda de dispositivos, especialmente el cepillo de dientes. Con la evolución tecnológica, se empezaron a fabricar cepillos de dientes con diferentes materiales. El objetivo fue investigar las marcas y modelos de cepillos de dientes disponibles en el mercado brasileño; analizar la legislación vigente en materia de producción y verificar la información proporcionada por el fabricante al consumidor final. Se trata de un estudio transversal, descriptivo y cualitativo de análisis documental. Se realizó una encuesta en las páginas electrónicas de la Agencia Nacional de Vigilancia Sanitaria y del Instituto Nacional de Metrología, Calidad y Tecnología sobre la legislación vigente, que establece las características requeridas para la producción y comercialización de cepillos de dientes. Buscamos marcas y modelos disponibles en el mercado brasileño, en los sitios web de empresas, fabricantes o importadores y en puntos de venta para que el consumidor final revise la información disponible en el empaque. La Resolución $\mathrm{N}^{\circ} 54 / 17$ de la Junta Colegiada de ANVISA establece algunas características físicas, tales como: ancho máximo de cabeza y largo mínimo del cepillo de dientes. En esta investigación se incluyeron veinte marcas comerciales, que distribuyen 108 modelos en el mercado brasileño. Solo 2 modelos $(1,85 \%)$ presentaron toda la información requerida por la resolución de ANVISA, siendo "supervisión de un adulto" y "orientación del dentista" los ítems menos contemplados. El 44,4\% del total no tenía indicación para uso adulto o infantil. En conclusión, hay muchas marcas y modelos en el mercado brasileño, pero falta información importante para que el profesional realice la indicación adecuada y el paciente para la adquisición consciente.

Descriptores: Cepillado Dental; Higiene Bucal; Salud Bucal.

\section{INTRODUÇÃO}

O cuidado com o corpo constitui um importante fator cultural e social no desenvolvimento humano e está diretamente relacionado às condições de saúde geral, já que a doença periodontal e a cárie são umas das duas doenças crônicas mais comuns, além de serem as maiores causadoras de perda dental $^{1,2}$.

A higiene bucal, parte integrante dos cuidados corporais quando executada de forma correta, com o auxílio de instrumentos adequados, evita o acúmulo de biofilme na superfície dental. Fator inerente no desenvolvimento de doenças bucais como a gengivite, periodontite, cárie e até mesmo a perda precoce do elemento dental ${ }^{3}$.

Para a higiene bucal, há muito tempo, são empregados instrumentos ou dispositivos, em especial, a escova dentária. Historicamente, na era primitiva, esfregavam-se pedaços de gravetos nas superfícies dentárias, como revelaram os achados em 
tumbas etruscas e egípcias. Segundo manuscritos encontrados nas escavações de Ur, na Babilônia, em 3.500 a.C., palitos de ouro também eram utilizados para promover a limpeza dos dentes ${ }^{4}$. Desde então, os dispositivos utilizados com a finalidade de higienizar os dentes sofreram mudanças de acordo com a cultura de cada povo, até que em 100 d.C., Plínio, o Jovem, estabeleceu conceitos sobre o material utilizado para a confecção da primeira escova dentária, com cerdas de porco espinho ${ }^{5}$. Após essa época, foram propostos vários tipos de escovas: com cerdas de crina ou cauda de cavalo, com pelo de porco montadas em cabos feitos de osso bovino ou marfim feita pelos chineses ${ }^{5}$. Pierre Fauchard, pai da moderna odontologia, em 1728, era contra instrumentos de metal e preconizava o uso de esponjas umedecidas ou com preparado de ervas e raízes ${ }^{5}$.

A primeira patente industrial de escova dental americana foi registada em nome de Wadsworth, em 1857, nos Estados Unidos, iniciando seu processo de produção em massa no final do século XIX e com o avanço tecnológico, as cerdas naturais foram substituídas por materiais sintéticos, como exemplo o nylon ${ }^{1}$.

Ao longo dos anos, com a evolução tecnológica, surgiram novos equipamentos $\mathrm{e}$ materiais, e atualmente, diante da quantidade de produtos disponíveis no mercado e apelo mercadológico com estratégias de marketing empregadas pelas empresas, o consumidor acha-se confuso no momento da escolha e aquisição do produto.

Muitos estudos em escovas dentárias sobre desgaste das cerdas ${ }^{6-8}$, acondicionamento ${ }^{6,9}$ e modelos ${ }^{10-12}$ foram realizados, contudo, as informações são pouco empregadas pelos profissionais para indicação ou orientação de seus pacientes na prática clínica, bom como a população, também, em sua maioria não dispõe destas informações.

Informações sobre a especificação, tempo de uso, acondicionamento e a conservação da escova são importantes, entretanto, a população, em sua maioria, não dispõe destas informações. Assim, objetivou-se nesta pesquisa analisar a legislação vigente sobre características da escova dentária, investigar as marcas e modelos disponíveis no mercado brasileiro, bem como verificar as informações fornecidas pelo fabricante para o consumidor final.

\section{MATERIAL E MÉTODO}

Trata-se de um estudo transversal, descritivo, qualitativo, de análise documental. Foram realizadas buscas na página eletrônica da Agência Nacional de Vigilância Sanitária - ANVISA para obtenção de documentos oficiais, das Resoluções da Diretoria Colegiada (RDC) da legislação vigente e informações disponíveis relacionadas às características de escovas dentárias. Também foram coletados na página eletrônica da ANVISA documentos sobre a legislação vigente que normatizam a fabricação e a venda de escovas dentárias no mercado brasileiro.

Consultas às páginas eletrônicas dos fabricantes/importadores de escovas dentárias foram realizadas para obtenção dos modelos comercializados. Também foram verificadas informações impressas nas embalagens e em pontos de vendas direta ao consumidor. Para a análise das informações nas embalagens foram incluídos os modelos encontrados em diferentes pontos de vendas diretas ao consumidor, como supermercados, farmácias e lojas de conveniência, tendo em vista que nem todas as marcas disponibilizaram fotos das embalagens nas próprias páginas eletrônicas, a recente mudança na legislação e também a preocupação com a coleta de informações em páginas desatualizadas.

As variáveis analisadas foram: indicação de público alvo (uso adulto ou infantil); rigidez das cerdas; tipo de cabeça; tipo de uso (elétrico ou manual); atendimento às portarias (informações ao consumidor constantes na embalagem). Foram excluídos os modelos que não apresentaram informações quanto à idade também foi analisado se a escova dentária é indicada para fins específicos, tais como: interdental, para dentadura, pós cirúrgica, ortodôntica, interdental e para higiene da língua.

As variáveis "infantil" e "supervisão do adulto" contidas na Resolução da Diretoria Colegiada - RDC n ${ }^{\circ} 142$, de 17 de março de $2017^{13}$ são itens exclusivos para escovas destinadas ao público infantil, por isso o tamanho da amostra é menor quando comparado aos modelos indicados para o adulto.

\section{RESULTADOS}

Nas buscas realizadas nas páginas eletrônicas dos fabricantes e foram encontradas as seguintes marcas: Colgate ${ }^{\circledR}$, Oral-B ${ }^{\circledR}$, Aquafresh ${ }^{\mathrm{TM}}$, Tepe ${ }^{\circledR}$, Dental Clean, Curaprox, Johnson \& Johnson's, Condor, Sanfill ${ }^{\circledR}$, Kess, Bitufo ${ }^{\circledR}$, Ultra ${ }^{\circledR}$, Boni, Techline ${ }^{\circledR}$, Tek, Sorriso, Inova, Dentil, Jadefrog e Topz. Nos pontos de venda direta ao consumidor final, foram encontradas 11 marcas, que distribuem 108 modelos e observou-se que a maioria $(55,56 \%)$ das escovas dentárias encontradas apresentam em suas embalagens a indicação do público-alvo (adulto ou infantil), em conformidade com a Resolução da Diretoria Colegiada - RDC N97/96, da ANVISA. (Tabela 1)

A Resolução da Diretoria Colegiada - RDC $\mathrm{n}^{\mathrm{o}} 142$, de 17 de março de $2017^{13}$, da ANVISA em seu Artigo 19, da Seção IV, do Capítulo IV, determina que as embalagens devem apresentar: “...a indicação de substituição da escova a cada 3 (três) 
meses após iniciar o uso ou conforme orientação do dentista; a indicação de que o produto não é perecível, em substituição a indicação do prazo de validade, ou indicação do prazo de validade, se aplicável; para produtos infantis; a indicação de uso infantil, a apresentação da faixa etária a que se destinam e a indicação de que o uso deve ser supervisionado por adulto; a indicação de que o tipo de escova deve ser orientada pelo dentista; a indicação quanto à rigidez da área encerdada; e cuidados de conservação e local de armazenamento após o uso...". Notou-se que algumas marcas não apresentaram todas as informações exigidas pela resolução.

Tabela1: Distribuição numérica dos modelos de escovas dentais encontradas no Brasil, segundo indicação de público alvo no ano de 2018

\begin{tabular}{lcrr}
\multicolumn{4}{c}{ Modelos encontrados } \\
Indicação de uso & Classificação & $\mathrm{n}$ & $\%$ \\
\hline Adulto & Manual & 34 & $31,48 \%$ \\
\hline Infantil & Manual & 26 & $24,07 \%$ \\
\hline Sem indicação & Manual & 48 & $44,44 \%$ \\
\hline Total & & 108 & $100,00 \%$ \\
\hline Fonte: Dados da Pesquisa & & &
\end{tabular}

Do total de 108 modelos encontrados, apenas $2(1,85 \%)$ apresentaram todos os itens exigidos pela Resolução $142 / 17^{13}$ da ANVISA. Dentre os achados as variáveis "orientação do dentista" $n=13(12,04 \%)$ e "supervisão do adulto" $n=13$ (17,57\%) (Tabela 2) foram pouco contemplados, estando em desacordo com a legislação.

Tabela 2: Distribuição das escovas dentarias, segundo exigências dispostas na RDC $142 / 17$ da ANVISA. no ano de 2018

\begin{tabular}{|c|c|c|c|}
\hline Variável & & n & $\%$ \\
\hline \multirow{2}{*}{ Substituir a cada 3 meses } & sim & 97 & $89,81 \%$ \\
\hline & não & 11 & $10,19 \%$ \\
\hline \multirow{2}{*}{ Produto não perecível } & sim & 106 & $98,15 \%$ \\
\hline & não & 2 & $1,85 \%$ \\
\hline \multirow{2}{*}{ Faixa etária } & sim & 55 & $50,93 \%$ \\
\hline & não & 53 & $49,07 \%$ \\
\hline \multirow{2}{*}{ Orientação do CD } & $\operatorname{sim}$ & 13 & $12,04 \%$ \\
\hline & não & 95 & $87,96 \%$ \\
\hline \multirow{2}{*}{ Cerdas } & sim & 108 & $100,00 \%$ \\
\hline & não & 0 & $0,00 \%$ \\
\hline \multirow{2}{*}{ Conservação } & $\operatorname{sim}$ & 22 & $20,37 \%$ \\
\hline & não & 86 & $79,63 \%$ \\
\hline \multirow{2}{*}{ Infantil } & $\operatorname{sim}$ & 26 & $24,07 \%$ \\
\hline & não & 82 & $75,93 \%$ \\
\hline \multirow{2}{*}{ Supervisão do adulto } & $\operatorname{sim}$ & 13 & $12,04 \%$ \\
\hline & não & 95 & $87,96 \%$ \\
\hline
\end{tabular}

DISCUSSÃO

Neste estudo sobre as marcas e modelos de escovas dentárias disponíveis no mercado brasileiro, legislação vigente e as informações fornecidas pelo fabricante para o consumidor final, observou-se várias distorções entre a normativa vigente $\mathrm{e}$ as informações recebidas pelo consumidor.

$\mathrm{Na}$ normativa $\mathrm{N}^{\circ} 97 / 96$ da ANVISA, são estabelecidos como componentes da escova dentária: a cabeça (parte ativa da escova na qual estão localizadas as cerdas), o pescoço (extensão do cabo à qual a cabeça está unida) e o cabo (região da escova com a qual é feita a empunhadura $)^{14}$. A ANVISA, através da RDC $n^{\circ} 142^{13}$, define que as escovas são classificadas de acordo com a faixa etária e a rigidez dos tufos das cerdas. A faixa etária é normatizada pela classificação do comprimento da escova e da largura da cabeça, e a rigidez do tufos pela Medida de Rigidez da Área Encerdada, conforme a Norma ISO $8627^{15}$.

Existem numerosas opções de escova no mercado brasileiro para escolha do consumidor, entretanto, faltam informações importantes nas embalagens, tanto para o profissional indicar um modelo adequado ao seu paciente, quanto para o consumidor fazer a escolha que necessita. Segundo dados do INMETRO, há desconformidade nas informações destinadas ao consumidor ${ }^{16}$, assim como, a rotulagem, que representa um item importante não apenas para o consumidor, mas também para o fabricante ou importador, pois necessitam das informações na embalagem para que seus produtos sejam rastreados no mercado ${ }^{16}$. Diante do cenário estabelecido, o INMETRO recomenda, que a embalagem contenha informações sobre a rigidez das cerdas (macia, média e dura), nome e endereço do fornecedor, número do lote $\mathrm{e}$ informações de usabilidade como a durabilidade (tempo útil, qualidade do cabo, acabamento das extremidades das cerdas) ${ }^{16}$.

A ANVISA define a embalagem como o recipiente, pacote ou o envoltório para proteger, armazenar e facilitar o transporte de produtos ${ }^{13}$. Estudos sobre rotulagem mostram que além de ser um envoltório, elas são utilizadas como "vendedores mudos"17, utilizando-se do valor que a marca possui para atrair o consumidor, que em caso de dúvida entre produtos semelhantes é atraído por uma embalagem mais atrativa ${ }^{18}$. No ano de 2010, Bottan $^{19}$ estudou os critérios utilizados pelos consumidores para a escolha da escova dental, e identificou entre os entrevistados que $74,5 \%$ das mulheres e $74,6 \%$ dos homens não receberam informações do cirurgiãodentista sobre os critérios para a escolha da escova, sendo apenas $10,5 \%$ mencionou que a indicação do dentista era um critério de escolha ${ }^{19}$.

A maioria dos modelos encontrados no mercado brasileiro são manuais. Estudos demonstraram que a escova elétrica é mais eficaz na remoção da placa bacteriana e diminuição da gengivite $^{20-22}$, principalmente em áreas de difícil acesso, como a face lingual ${ }^{23}$. Outro estudo evidenciou a superioridade da escova elétrica na remoção da placa e diminuição da gengivite a curto e a longo prazo, quando comparada com a escova manual $^{20}$. Em um estudo realizado com escova de cabeça tripla, ficou demonstrado sua superioridade no controle do biofilme dentário em superfícies lisas de dentes posteriores, quando comparada com a convencional $^{24}$ tendo sido considerada a mais indicada para pacientes que necessitam de cuidadores $^{25}$. Ensaios clínicos sobre eficácia de escovas manuais concluíram, que não existe 
superioridade entre os modelos de dispositivos manuais $^{26-28}$.

O tempo de uso das escovas para sua substituição é controverso tanto na literatura quanto entre os profissionais e consumidores. Na RDC da ANVISA $\mathrm{N}^{\circ} 142 / 17^{13}$, determina que o fabricante deve indicar na embalagem a substituição da escova a cada 3 (três) meses após iniciar o uso ou conforme orientação do dentista ${ }^{13}$. Nota-se, entretanto, em estudos clínicos com escovas dentárias elétricas ${ }^{29}$, manuais de diferentes modelos que não houve significância estatística entre o desgaste da cerda e a idade da escova com a diminuição da eficácia na eliminação da placa ${ }^{29,30}$.

CONCLUSÃO

Existem muitas marcas e modelos no mercado brasileiro porém faltam informações importantes para o profissional realizar a indicação apropriada para o paciente. Também observou-se que tanto as escovas elétricas quanto as manuais são eficazes na remoção da placa bacteriana, sendo o fator "preço" altamente associado à escolha do consumidor.

\section{REFERENCIAS}

1. Jardim JJ, Alves LS, Maltz M. The history and global market of oral home-care products. Braz Oral Res. 2009;23(Suppl 1):17-22.

2. Fischman SL. The history of oral hygiene products: how far have we come in 6000 years? Periodontol 2000. 1997;15:7-14.

3. Borghi WMMC, Moimaz SAS, Saliba NA. Métodos alternativos para higienização bucal e terapêutica odontológica. Rev Inst Ciênc Saúde. 2005;23(4):309-14.

4. McCauley HB. Toothbrushes, toothbrush materials and design. J Am Dent Assoc. 1946;33(5):283-93.

5. Garfin LA. Tooth picks and tooth brushes. Dent Survery. 1964;40:102-8.

6. Garbin CAS, Rovida TAS, Garbin AJÍ, Arcieri RM. Saúde bucal e educação infantil: avaliação do desgaste e do acondicionamento de escovas dentárias utilizadas por pré-escolares. Rev Odontol UNESP. 2012;41:148.

7. Conforti NJ, Cordero RE, Liebman J, Bowman JP, Putt MS, Kuebler DS, et al. An investigation into the effect of three months' clinical wear on toothbrush efficacy: results from two independent studies. J Clin Dent. 2003;14(2):29-33.

8. Kaiser E, Meyners M, Markgraf D, Stoerkel U, von Koppenfels R, Adam R, et al. Brush head composition, wear profile, and cleaning efficacy: an assessment of three electric brush heads using in vitro methods. J Clin Dent. 2014;25(2):19-25.

9. R aiyani CM, Arora R, Bhayya DP, Dogra S, Katageri AA, Singh V. Assessment of microbial contamination on twice a day used toothbrush head after 1-month and 3 months: an in vitro study. J Nat Sci Biol Med. 2015;6(Suppl 1):S44-8.

10. Hamal JD, Hensley DM, Maller SC, Palazzolo DJ, Vandewalle KS. An in vitro comparison of antimicrobial toothbrushes. Gen Dent. 2014;62(6):e24-7.

11. Slot DE, Wiggelinkhuizen L, Rosema NA, Van der Weijden GA. The efficacy of manual toothbrushes following a brushing exercise: a systematic review. Int $\mathrm{J}$ Dent Hyg. 2012;10(3):187-97.

12. Niazi F, Naseem M, Khurshid Z, Zafar MS, Almas K. Role of Salvadora persica chewing stick (miswak): A natural toothbrush for holistic oral health. Eur J Dent. 2016;10(2):301-8.

13. Brasil. Ministério da Saúde. Resolução - RDC no 142, de 17 de março de 2017. Dispõe sobre a regularização de produtos de higiene pessoal descartáveis destinados ao asseio corporal, que compreendem escovas e hastes para higiene bucal, fios e fitas dentais, absorventes higiênicos descartáveis, coletores menstruais e hastes flexíveis. Diário Oficial União. 2017 mar20.

14. Brasil. Ministério da Saúde. Portaria no 97, de 26 de junho de 1996. Dispõe sobre as Normas e Requisitos Técnicos, a que ficam sujeitos as escovas dentais, com ou sem pigmentos ou corantes nas cerdas. Diário Oficial União. 1996 jun 27.

15. International Organization for Standardization. ISO 8627:1987: dentistry: stiffness of the tufted area of tooth-brushes. Disponível em: https://www.iso.org/standard/15961.html.

16. Brasil. Ministério do Desenvolvimento, Indústria e Comércio Exterior. Programa de análise de produtos: relatório da análise em escovas dentais para uso adulto e infantil. Disponível em: http://estaticog1.globo.com/2013/06/10/rel_escov as_dentais_final.pdf.

17. Pontes TE, Costa TF, Marum ABRF, Brasil ALD, Taddei JAAC. Orientação nutricional de crianças e adolescentes e os novos padrões de consumo: propagandas, embalagens e rótulos. Rev Paul Pediatr. 2009;27(1):99-105.

18. Associação Brasileira de Embalagem. Valor do design. Disponível em: http://www.abre.org.br/comitesdetrabalho/design/ valor-do-design/

19. Bottan ER, Campos L, Odebrecht CMLR, Silveira EG, Schmitt P, Araújo SM. Critérios adotados para a escolha da escova dental: estudo com consumidores de Florianópolis, Santa Catarina (Brasil). RSBO Rev Sul-Bras Odontol. 2010;7(2):173-81.

20. Yaacob M, Worthington HV, Deacon SA, Deery C, Walmsley AD, Robinson PG, et al. Powered versus manual toothbrushing for oral health. Cochrane Database Syst Rev. 2014;(6):CD002281. 
21. Vorwerk L, Ghassemi A, Hooper W, Patel V, Milleman J, Milleman K. Comparative plaque removal efficacy of a new powered toothbrush and a manual toothbrush. J Clin Dent. 2016;27(3):76-9.

22. Li Z, He T, Li C, Sun L, Chang J, He Y, et al. A randomized 3-month clinical comparison of a power toothbrush to a manual toothbrush in the reduction of gingivitis. Am $\mathrm{J}$ Dent. 2016;29(4):193-6

23. Costa CC, Costa Filho LC, Sória ML, Mainardi APR. Plaque removal by manual and electric toothbrushing among children. Pesqui Odontol Bras. 2001;15(4):296-301.

24. Oliveira LB, Zardetto CGDC, Rocha RO, Rodrigues CRMD, Wanderley MT. Eficácia da escova dental convencional e de cabeça tripla na remoção do biofilme dental em crianças. RGO, Rev Gaúch Odontol. 2011;59(2):259-63.

25. Kalf-Scholte SM, Van der Weijden GA, Bakker EWP, Slot DE. Plaque removal with triple-headed vs single-headed manual toothbrushes: a systematic review. Int J Dent Hyg. 2018;16(1):13-23

26. Aass AM, Gjermo P. Comparison of oral hygiene efficacy of one manual and two electric toothbrushes. Acta Odontol Scand. 2000;58(4):166-70.

27. Laher A, Kroon J, Booyens SJ. Effectiveness of four manual toothbrushes in a cohort of patients undergoing fixed orthodontic treatment in an academic training hospital. SADJ. 2003;58(6):231-37.

28. Gomes LK, Sarmento CF, Seabra FRG, Santos PBD, Pinheiro FHSL. Randomized clinical controlled trial on the effectiveness of conventional and orthodontic manual toothbrushes. Braz Oral Res. 2012;26(4):360-65.

29. Hogan LME, Daly CG, Curtis BH. Comparison of new and 3-month-old brush heads in the removal of plaque using a powered toothbrush. J Clin Periodontol. 2007;34(2):130-36.

30. Rosema NA, Hennequin-Hoenderdos NL, Versteeg PA, van Palenstein Helderman WH, van der Velden U, et al. Plaque-removing efficacy of new and used manual toothbrushes: a professional brushing study. Int J Dent Hyg. 2013;11(4):237-43.

\section{CONFLITO DE INTERESSES}

Os autores declaram não haver conflitos de interesse.

\section{AUTOR PARA CORRESPONDÊNCIA}

\section{Suzely Adas Saliba Moimaz}

Programa de Pós-Graduação em Saúde Coletiva em Odontologia Núcleo de Pesquisa em Saúde Coletiva, NEPESCO

Rua José Bonifácio, 1193 - Vila Mendonça

16015-050 Araçatuba- SP, Brasil

E-mail: suzely.moimaz@unesp.br
Submetido em 19/07/2019

Aceito em 05/09/2019 